\title{
Combined effects of perturbations, radiation and oblateness on the periodic orbits in the restricted three-body problem
}

\author{
AbdulRazaq AbdulRaheem · Jagadish Singh
}

Received: 6 May 2008 / Accepted: 26 May 2008 / Published online: 19 July 2008

(C) The Author(s) 2008. This article is published with open access at Springerlink.com

\begin{abstract}
This paper investigates the periodic orbits around the triangular equilibrium points for $0<\mu<\mu_{c}$, where $\mu_{c}$ is the critical mass value, under the combined influence of small perturbations in the Coriolis and the centrifugal forces respectively, together with the effects of oblateness and radiation pressures of the primaries. It is found that the perturbing forces affect the period, orientation and the eccentricities of the long and short periodic orbits.
\end{abstract}

Keywords Oblateness - Radiation - Equilibrium points . Coriolis force $\cdot$ Centrifugal force $\cdot$ Restricted three-body problem $\cdot$ Periodic orbits

\section{Introduction}

The restricted three-body problem has five equilibrium points, two triangular and three collinear. The collinear points $\mathrm{L}_{1}, \mathrm{~L}_{2}, \mathrm{~L}_{3}$ are unstable for any value of mass ratio $\mu$ of the smaller primary to the total mass of the primaries, while the triangular points $\mathrm{L}_{4}, \mathrm{~L}_{5}$ are stable for $0<\mu<\mu_{c}$, where $\mu_{c}$ is the critical mass value of the mass ratio $\mu$ of the finite bodies (Szebehely 1967).

Due to the rotational motion, periodic orbits exist in the restricted three-body problem, long and short periodic orbits are found around the equilibrium points. Elements that could be used to describe the motion of an infinitesimal body relative to the primaries are categorized as orbital and non orbital elements. Angular momentum and total energy are

A. AbdulRaheem $(\bowtie) \cdot$ J. Singh

Department of Mathematics, Faculty of Science, Ahmadu Bello

University, Zaria, Nigeria

e-mail: raz11ng@yahoo.com the integrals available to measure the shapes and sizes of the orbits but are not directly observable. Therefore, eccentricities, inclination and semi major axes of the orbits are used to determine the shapes, orientation and sizes of the orbits.

In recent times perturbing forces such as oblateness and radiation forces of the primaries as well as the Coriolis and centrifugal forces have been considered variously in the studies related to the equilibrium points of the restricted three-body problem. Sharma (1987) studied the linear stability of the restricted problem in which the bigger primary is a source of radiation and the smaller primary an oblate spheroid. He proved that the collinear equilibrium have conditional retrograde elliptical periodic orbits around them, while the triangular points have long or short periodic retrograde elliptical orbits for the mass parameter $\mu$ such that $0<\mu<\mu_{c}$. He further showed that the eccentricity of the retrograde elliptical periodic orbits increases with oblateness and decreases with radiation forces.

By using second order parametric expansions, the families of periodic orbits generated from the inner collinear equilibrium point in a binary system were numerically determined by Ragos et al. (1991). Long and short periodic solutions with similar character of periodicity of $\mathrm{L}_{4}$ were identified. It was also found that finite periodic solutions in the vicinity of $\mathrm{L}_{1}$ are stable.

In the case of restricted three-body problem in which the primaries are triaxial rigid bodies, Sharma et al. (2001) observed that the triangular points have long and short periodic elliptical orbits for $\mu$ such that $0<\mu<\mu_{c}$. Ishwar and Elipe (2001) obtained the secular solutions of the triangular equilibrium points in the restricted three-body problem in which the smaller primary is an oblate spheroid and the more massive primary is a source of radiation. They found that the triangular points have long and short periodic retrograde elliptical orbits. 
In a recent paper (AbdulRaheem and Singh 2006), the stability of equilibrium points of the restricted three-body problem under the combined influence of small perturbations in the Coriolis and the centrifugal forces, together with the effects of oblateness and radiation of the primaries were investigated. It was found that the range of stability of the triangular points decreases for $\mu$ such that $0<\mu<\mu_{c}$ while the collinear points remain unstable.

In this paper, using the same assumptions, results and parameters as in our aforesaid paper of 2006, we study the periodic solutions of the generalized problem around the triangular equilibrium point $\mathrm{L}_{4}$ within the stable region $\left(0<\mu<\mu_{c}\right)$. Thereafter, we characterize the periodic orbits to be elliptical. Further, we describe the eccentricities and the orientations of the periodic orbits.

\section{Equations of motion}

Let $m_{1}$ and $m_{2}$ denote the masses of the bigger and smaller primaries respectively, and $\mathrm{m}$ the mass of the infinitesimal body. Let $A_{1}$ and $A_{2}$ denote the oblateness coefficients of the bigger and smaller primaries respectively such that $0<A_{i} \ll 1,(i=1,2)$. Further, we denote the respective radiation factors for the bigger and smaller primaries as $q_{i}$ $(i=1,2)$ such that $0<1-q_{i} \ll 1,(i=1,2)$. Let the parameters $\varphi$ and $\psi$ denote the Coriolis and centrifugal forces, arising due to rotational motion, respectively. We denote the small perturbations in the Coriolis and centrifugal forces by $\varepsilon$ and $\varepsilon^{\prime}$ respectively such that $\varphi=1+\varepsilon,|\varepsilon| \ll 1$ and $\psi=1+\varepsilon^{\prime},\left|\varepsilon^{\prime}\right| \ll 1$.

Let $(x, y)$ be the coordinates of the infinitesimal body in the orbital plane. Following the notations and terminology of Szebehely (1967), the equations of motion of the infinitesimal body in the dimensionless barycentric-synodic coordinate system (AbdulRaheem and Singh 2006) are

$\ddot{x}-2 n \varphi \dot{y}=\Omega_{x}$,

$\ddot{y}+2 n \varphi \dot{x}=\Omega_{y}$,

where the potential is

$$
\begin{aligned}
\Omega= & \frac{1}{2} n^{2} \psi\left(x^{2}+y^{2}\right)+\frac{1-\mu}{r_{1}} q_{1}+\frac{\mu}{r_{2}} q^{2} \\
& +\frac{1-\mu}{2 r_{1}^{3}} A_{1} q_{1}+\frac{\mu}{2 r_{2}^{3}} A_{2} q_{2},
\end{aligned}
$$

the distances of the primaries from the infinitesimal body are

$r_{1}^{2}=(x-\mu)^{2}+y^{2}$,

$r_{2}^{2}=(x+1-\mu)^{2}+y^{2}$ and $n$, the perturbed mean motion of the primaries is given by

$n^{2}=1+\frac{3}{2}\left(A_{1}+A_{2}\right)$.

Equations of motion (1) admits the Jacobi integral

$\dot{x}^{2}+\dot{y}^{2}-2 \Omega+C=0$,

where $C$ is the Jacobian constant.

\section{Periodic orbits}

We denote the equilibrium points and their positions as $L\left(x_{0}, \pm y_{0}\right)$. Let a small displacement in $\left(x_{0}, y_{0}\right)$ be $(\xi, \eta)$. Then the corresponding characteristic equation of our problem is (AbdulRaheem and Singh 2006)

$\lambda^{4}-\left(\Omega_{x x}^{0}+\Omega_{y y}^{0}-4 n^{2} \varphi^{2}\right) \lambda^{2}+\Omega_{x x}^{0} \Omega_{y y}^{0}-\left(\Omega_{x y}^{0}\right)^{2}=0$,

where, the superscript 0 indicates that the partial derivatives of the potential $\Omega$ are evaluated at the equilibrium point $\mathrm{L}_{4}$. In evaluating the partial derives we restrict ourselves to linear terms in $\varepsilon$ and $\varepsilon^{\prime}$, and neglect their products with $A_{i}$ and $1-q_{i}(i=1,2)$.

In the stable region $0<\mu<\mu_{c}$, the characteristic equation (6) has pure imaginary roots. Hence the motion is bounded and is composed of two harmonic motions given by

$\xi=C_{1} \cos s_{1} t+S_{1} \sin s_{1} t+C_{2} \cos s_{2} t+S_{2} \sin s_{2} t$,

$\eta=\bar{C}_{1} \cos s_{1} t+\bar{S}_{1} \sin s_{1} t+\bar{C}_{2} \cos s_{2} t+\bar{S}_{2} \sin s_{2} t$,

with different frequencies $s_{1}$ and $s_{2}$, where

$$
\begin{aligned}
s_{1}= & {\left[\frac{27}{4} \mu(1-\mu)+\frac{33}{2} \mu(1-\mu) \varepsilon^{\prime}+\frac{3}{2} \mu(1-\mu)\left(1-q_{1}\right)\right.} \\
& +\frac{3}{2} \mu(1-\mu)\left(1-q_{2}\right)+\frac{45}{2} \mu\left(1-\frac{13}{10} \mu\right) A_{1} \\
& \left.+\frac{117}{4} \mu(1-\mu) A_{2}\right]^{\frac{1}{2}}
\end{aligned}
$$

and

$$
\begin{aligned}
s_{2}= & -\frac{27}{8} \mu(1+\mu)+4 \varepsilon-\frac{3}{2}\left(1+\frac{11}{2} \mu\right) \varepsilon^{\prime}-\frac{33}{4} \mu^{2} \varepsilon^{\prime} \\
& -\frac{3}{4} \mu(1+\mu)\left(1-q_{1}\right)-\frac{3}{4} \mu(1+\mu)\left(1-q_{2}\right) \\
& -\frac{3}{4}(1+13 \mu) A_{1}+\frac{3}{4} \mu\left(1-\frac{43}{2} \mu\right) A_{2} \\
& -\frac{117}{8} \mu^{2} A_{1}-\frac{117}{8} \mu^{2} A_{2} .
\end{aligned}
$$


The frequency $s_{1}$ is for the long periodic orbit while $s_{2}$ is for the short periodic orbit. The coefficients $C_{1}, S_{1}, \bar{C}_{1}$ and $\bar{S}_{1}$ are the long periodic terms while $C_{2}, S_{2}, \bar{C}_{2}$ and $\bar{S}_{2}$ are the short periodic terms.

\section{Elliptical orbits}

The expansion of $\Omega$ around the triangular point $\mathrm{L}_{4}$ is

$\Omega=\Omega^{0}+\frac{1}{2} \Omega_{x x}^{0} \xi^{2}+\Omega_{x y}^{0} \xi \eta+\frac{1}{2} \Omega_{y y}^{0} \eta^{2}+O(3)$.

It can be shown that (9) is a quadratic form and indicates that the periodic orbits around $\mathrm{L}_{4}$ are elliptical.

\subsection{Orientation}

Equation (9) can be expressed in the form

$\Omega=L \xi^{2}+M \xi \eta+N \eta^{2}+U$,

where

$$
\begin{aligned}
L= & \frac{3}{8}+\frac{5}{8} \varepsilon^{\prime}-\frac{1}{4}(1-3 \mu)\left(1-q_{1}\right) \\
& +\frac{1}{2}\left(1-\frac{3}{2} \mu\right)\left(1-q_{2}\right)+\frac{27}{16}\left(1-\frac{4}{9} \mu\right) A_{1} \\
& +\frac{3}{16}(1+8 \mu) A_{2}, \\
M= & \sqrt{3}\left[-\frac{3}{4}+\frac{3}{2} \mu-\frac{7}{6}(1-2 \mu) \varepsilon^{\prime}+\frac{1}{6}(1+\mu)\left(1-q_{1}\right)\right. \\
& -\frac{1}{3}\left(1-\frac{1}{2} \mu\right)\left(1-q_{2}\right)-\frac{19}{8}\left(1-\frac{26}{19} \mu\right) A_{1} \\
& \left.-\frac{7}{8}\left(1-\frac{26}{7}\right) A_{2}\right], \\
N= & \frac{9}{8}+\frac{7}{3} \varepsilon^{\prime}+\frac{3}{4}(1-3 \mu)\left(1-q_{1}\right) \\
& -\frac{1}{2}\left(1-\frac{3}{2} \mu\right)\left(1-q_{2}\right)+\frac{33}{16} A_{1}+\frac{33}{16} A_{2}, \\
U= & \frac{3}{2}+\frac{1}{2} \varepsilon^{\prime}-(1-\mu)\left(1-q_{1}\right)-\mu\left(1-q_{2}\right) \\
& +\frac{5}{4}\left(1-\frac{2}{5} \mu\right) A_{1}+\frac{3}{4}\left(1+\frac{2}{3} \mu\right) A_{2} .
\end{aligned}
$$

We introduce the variables $\bar{\xi}$ and $\bar{\eta}$ in (10) by the transformation

$$
\begin{gathered}
\xi=\bar{\xi} \cos \theta-\bar{\eta} \sin \theta, \\
\eta=\bar{\xi} \sin \theta+\bar{\eta} \cos \theta .
\end{gathered}
$$

This is equivalent to a rotation of the coordinate system $\xi, \eta$ through angle $\theta$. We choose $\theta$ such that the terms containing $\bar{\xi} \bar{\eta}$ in the equation equal to zero. The new quadratic form is given as

$\bar{\Omega}=\bar{L} \bar{\xi}^{2}+\bar{M} \bar{\eta}^{2}+\bar{U}$.

By setting the term containing $\bar{\xi} \bar{\eta}$ equal to zero we have

$$
\begin{aligned}
\tan \theta= & \sqrt{3}\left[1-2 \mu+\frac{11}{9}\left(1-\frac{28}{11} \mu\right) \varepsilon^{\prime}\right. \\
& -\frac{8}{9}(1-2 \mu)\left(1-q_{1}\right)+\frac{16}{9}\left(1-\frac{5}{4} \mu\right)\left(1-q_{2}\right) \\
& \left.+\frac{8}{3}\left(1-\frac{19}{8} \mu\right) A_{1}-\frac{4}{3}\left(1+\frac{7}{4} \mu\right) A_{2}\right]
\end{aligned}
$$

4.2 Eccentricities of the ellipses

The eccentricities are given by equations (Szebehely 1967)

$e_{i}=\left(1-\alpha_{i}^{2}\right)^{\frac{1}{2}}$,

where

$\alpha_{i}=\frac{2 s_{i}}{s_{i}^{2}+\bar{\lambda}} \quad(i=1,2)$.

The term $\bar{\lambda}$ is one the roots of the characteristic equation obtained from the relation $C=2 \bar{\Omega}$. For $i=1$, we have

$$
\begin{aligned}
\alpha_{1}^{2}= & 3 \mu-12 \mu^{2}+\frac{7}{12} \mu \varepsilon^{\prime}-\frac{79}{3} \mu^{2} \varepsilon^{\prime} \\
& +\frac{2}{3} \mu\left(1-q_{1}\right)+\frac{2}{3} \mu\left(1-q_{2}\right)-\frac{8}{3} \mu^{2}\left(1-q_{1}\right) \\
& -\frac{8}{3} \mu^{2}\left(1-q_{2}\right)-5 \mu A_{1}+4 \mu A_{2} \\
& -\frac{65}{2} \mu^{2} A_{1}-58 \mu^{2} A_{2}
\end{aligned}
$$

and

$$
\begin{aligned}
e_{1}= & 1-\frac{3}{2} \mu+6 \mu^{2}-\frac{7}{24} \mu \varepsilon^{\prime}-\frac{79}{6} \mu^{2} \varepsilon^{\prime} \\
& -\frac{1}{3} \mu\left(1-q_{1}\right)-\frac{1}{3} \mu\left(1-q_{2}\right)+\frac{4}{3} \mu^{2}\left(1-q_{1}\right) \\
& +\frac{4}{3} \mu^{2}\left(1-q_{2}\right)+\frac{5}{2} \mu A_{1}-2 \mu A_{2} \\
& +\frac{65}{4} \mu^{2} A_{1}+29 \mu^{2} A_{2} .
\end{aligned}
$$

For $i=2$, we have

$$
\begin{aligned}
\alpha_{2}^{2}= & \frac{1}{4}-\frac{9}{16} \mu-\frac{9}{16} \mu^{2}+\varepsilon+\left(-\frac{9}{8}-\frac{85}{16} \mu\right) \varepsilon^{\prime} \\
& -\frac{47}{16} \mu^{2} \varepsilon^{\prime}-\frac{1}{8} \mu\left(1-q_{1}\right)-\frac{1}{8} \mu\left(1-q_{2}\right) \\
& -\frac{1}{4} \mu^{2}\left(1-q_{1}\right)-\frac{1}{4} \mu^{2}\left(1-q_{2}\right) \\
& +\left(-\frac{1}{9}+\frac{51}{16} \mu\right) A_{1}+\left(-\frac{3}{8}-\frac{51}{16} \mu\right) A_{2} \\
& -\frac{39}{8} \mu^{2} A_{1}-\frac{39}{8} \mu^{2} A_{2}
\end{aligned}
$$


and

$$
\begin{aligned}
e_{2}= & \frac{\sqrt{3}}{2}\left[1+\frac{3}{8} \mu(1+\mu)-\frac{2}{3} \varepsilon+\frac{3}{4}\left(1+\frac{85}{18} \mu\right) \varepsilon^{\prime}\right. \\
& +\frac{47}{24} \mu^{2} \varepsilon^{\prime}+\frac{1}{12} \mu\left(1-q_{1}\right) \\
& +\frac{1}{12} \mu\left(1-q_{2}\right)+\frac{1}{6} \mu^{2}\left(1-q_{1}\right) \\
& +\frac{1}{6} \mu^{2}\left(1-q_{2}\right)+\frac{3}{4}\left(1-\frac{17}{6} \mu\right) A_{1} \\
& \left.+\frac{1}{2}\left(1+\frac{17}{4}\right) A_{2}+\frac{13}{4} \mu^{2} A_{1}+\frac{13}{4} \mu^{2} A_{2}\right]
\end{aligned}
$$

\section{Discussion}

It is clear from (8) that the small perturbations in the Coriolis and the centrifugal forces, radiation and oblateness of the primaries affect the long and short periods. For small $\mu$, the frequency $s_{1}$ of the long period increases with increments in the forces while $s_{2}$, the frequency of the short period deceases with increments in the same parameters. The implication is that the period of the long period orbit decreases while that of short period orbit increases. However, the two orbits coincide at the critical value of the mass ratio $\mu_{c}$, where the periods are equal (see Fig. 1).

In the absence of small perturbations in the Coriolis and the centrifugal forces, when the primaries are neither radiating nor oblate, our results agree with Szebehely's results (1967). In the absence of perturbations and radiations, we validate the results of Sharma et al. (2001). When there are no perturbations, the smaller primary is not radiating and the bigger primary is not oblate, we verify the results of Ishwar and Elipe (2001), and of Sharma (1987).

It is easily seen from (10) that the periodic orbits around $\mathrm{L}_{4}$ are elliptical. The orientation of the orbits with respect to the rotational coordinate system is determined. Equation (13) indicates that the perturbing forces affect the size of the angle of inclination of the orbits. For small $\mu$, an increase in the perturbing forces produces corresponding increase in the inclination angle of the orbits, thereby changing the orientation of the orbits along the $\bar{\xi}$ coordinate.

In the absence of small perturbation in the centrifugal force, when the primaries are neither radiating nor oblate, the orientation corresponds to the orientation of the classical problem obtained by Szebehely (1967). When there is no perturbation in the centrifugal force and primaries are not radiating, we verify the orientation of the problem of Sharma et al. (2001). In the absence of perturbation in the centrifugal force, when the bigger primary is radiating and the smaller primary is oblate, we validate the orientation of Ishwar and Elipe's problem (2001).

Equations (15) and (16) describe the eccentricities of the long and short periodic orbits around the point $\mathrm{L}_{4}$ respectively. The eccentricity of the long period orbit decreases with increments in small perturbation in the centrifugal force, radiations and oblateness coefficients of the primaries, while the eccentricity of the short period orbit increases with increments in the same parameters. The implication of this is that the ellipticity of the orbits is adjusted due to the effects of the perturbing forces. In fact, the two orbits coincide at the critical mass value $\mu_{c}$, and the eccentricities of the orbits are equal (see Fig. 2).

Both of them generalize the eccentricities of the classical problem (Szebehely 1967). For small $\mu$, when there are no perturbations in the Coriolis and the centrifugal forces, the
Fig. 1 Frequencies of the long and short periods, where series $1=s_{1}$ and series $2=s_{2}$

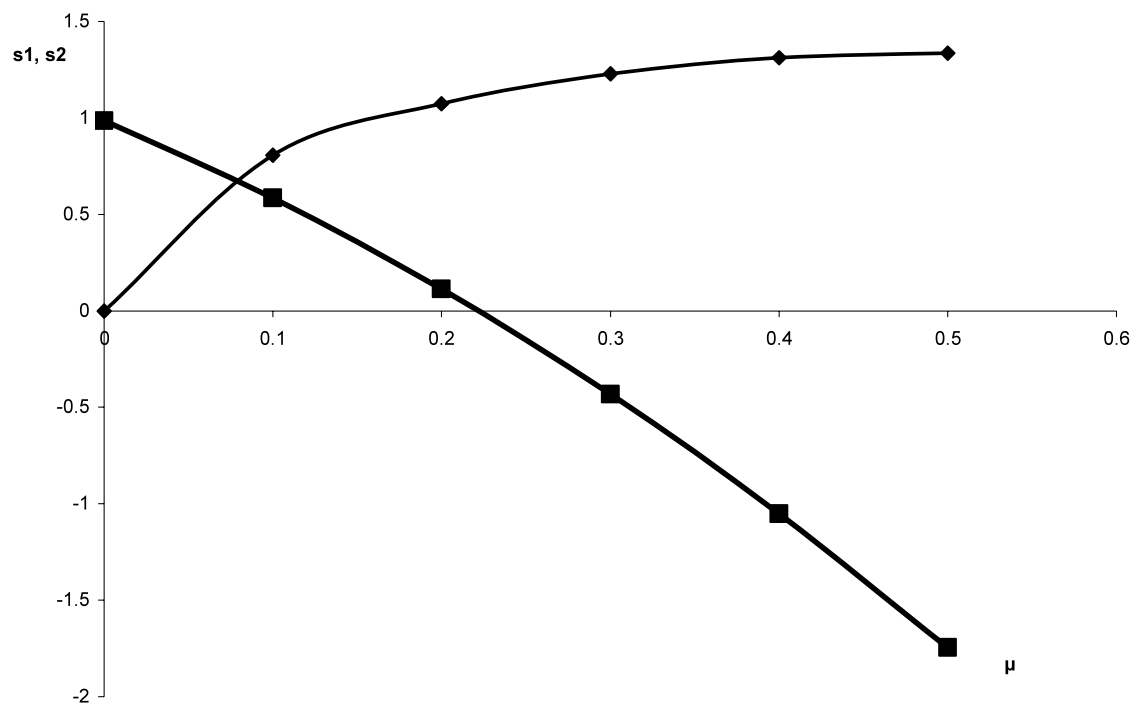

$\neg$ Series 1 
Fig. 2 Eccentricities of the long and short periodic orbits, where series $1=e_{1}$ and series $2=e_{2}$

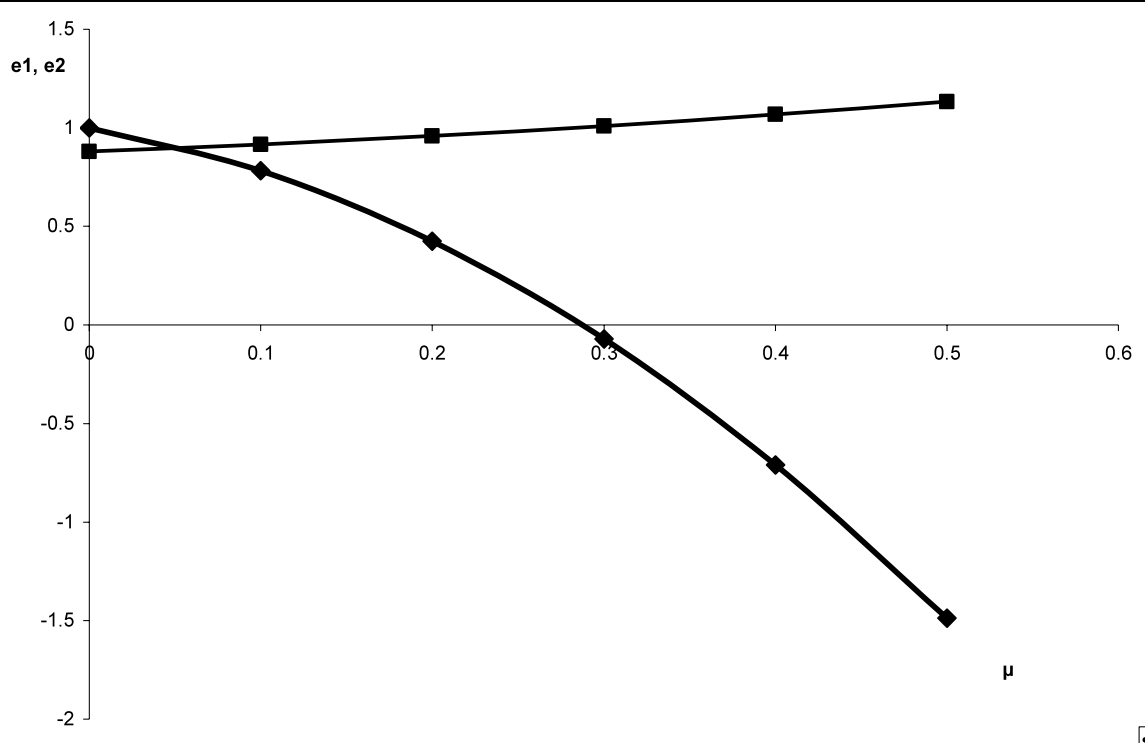

smaller primary is not radiating and the bigger primary is not oblate, our results correspond to the results of Ishwar and Elipe (2001).

\section{Conclusion}

Periodic orbits around the triangular equilibrium points $\mathrm{L}_{4}$ for $0<\mu<\mu_{c}$, where $\mu_{c}$ is the critical mass value, were studied under the combined influence of perturbations in the Coriolis and the centrifugal forces, radiations and oblateness of the primaries. It is observed that small increment in the perturbing forces causes decrease in the period of the long periodic orbit, while the period of the short periodic orbit increases. The two periods coincide at the critical mass value $\mu_{c}$. The effects of the small increment in the perturbing forces results in the shift of the inclination angle of the orbits thereby adjusting the orientation of the orbits. Again the small increment in the perturbing forces led to decrease in the eccentricity of the long periodic orbit while that of the short periodic orbit increases thereby adjusting the shapes of the orbits. Since the problem of space dynamics is how to construct satellites that will move around in the neighborhood of celestial bodies, it is hoped that results of this research will be useful for space engineers in sending satellites to stable regions.

Acknowledgements The authors are extremely grateful to Professor D. Singh and Professor G.U. Garba, the head of department of Mathematics, Ahmadu Bello University, Zaria for their encouragements.

Open Access This article is distributed under the terms of the Creative Commons Attribution Noncommercial License which permits any noncommercial use, distribution, and reproduction in any medium, provided the original author(s) and source are credited.

\section{References}

AbdulRaheem, A., Singh, J.: Astron. J. 131, 1880-1885 (2006)

Ishwar, B., Elipe, A.: Astrophys. Space Sci. 277, 437-446 (2001)

Ragos, O., et al.: Astrophys. Space Sci. 182, 313-316 (1991)

Sharma, R.K.: Astrophys. Space Sci. 185, 271-281 (1987)

Sharma, R.K., et al.: Celest. Mech. Dyn. Astron. 79, 119-133 (2001)

Szebehely, V.: Theory of Orbits. Academic Press, San Diego (1967) 